\section{Combining TNF blockade with immune checkpoint inhibitors in patients with cancer}

\author{
Anne Montfort, Mathieu Virazels, Céline Colacios, Nicolas Meyer and \\ Bruno Ségui(i)
}

TNF is involved in various autoimmune diseases and in immune-related adverse events (irAEs) that occur in patients with cancer being treated with immune checkpoint inhibitors (ICIs) ${ }^{1,2}$. In their Review (Chen, A. Y., Wolchok, J. D., \& Bass, A. R. TNF in the era of immune checkpoint inhibitors: friend or foe? Nat. Rev. Rheumatol. 17, 213-223 (2021) ) ${ }^{3}$, Chen and colleagues nicely reviewed the literature, from basic studies ${ }^{4,5}$ to clinical observations ${ }^{6,7}$, discussing whether TNF can be considered as a putative target in the treatment of irAEs in patients with cancer undergoing ICI therapy. Important questions were raised regarding TNF inhibitor safety and efficacy in this setting, but unfortunately, the authors missed out discussions of the TICIMEL phase Ib clinical trial (NTC03293784), the results of which we think help address some of these questions.

Initiated in 2018, the TICIMEL trial investigated the effects of treatment with the ICIs ipilimumab (an anti-CTLA4 antibody) and nivolumab (an anti-PD1 antibody) in combination with a TNF inhibitor (infliximab or certolizumab) in patients with advanced melanoma ${ }^{8}$. The results from 14 patients enrolled in the first phase of this trial were published in December 2020 (REF. ${ }^{9}$ ). Although the low number of patients warrants caution as regard to the interpretation of data, the results are informative.

One question raised by Chen and colleagues relates to whether TNF inhibitors are safe in the management of patients with cancer and ICI-induced irAEs. Results from the TICIMEL trial indicate that concomitant administration of ipilimumab, nivolumab and an anti-TNF drug (infliximab or certolizumab) is indeed safe in the short-term and potentially in the long-term.

Chen and colleagues also compiled evidence from pre-clinical studies showing that TNF promotes cancer progression and inhibits anti-tumour immune responses. They conclude that although TNF blockade and/or deficiency in mouse models of cancer can, via the promotion of $\mathrm{CD}^{+} \mathrm{T}$ cellmediated anti-tumour immune responses and a decrease in immune regulatory responses, impede tumour growth, these observations have to be confirmed in humans. Especially, they noted that this hypothesis has to be evaluated in the context of combined ICI and anti-TNF treatment.

In line with these observations, results from the TICIMEL trial show a high objective response rate in the certolizumab cohort, with all evaluable patients responding to treatment, including four complete responses out of seven objective responses. By comparison, only half of the patients in the infliximab cohort responded to treatment (including one complete response out of three objective responses). These treatments were associated with increased numbers of $\mathrm{T}$ helper 1 cells and increased plasma concentrations of IFN $\gamma$. Whether and how these responses differ to the ones occurring in patients with advanced melanoma being treated with the combination of ipilimumab and nivolumab remains to be evaluated.

Emerging evidence reported by Chen and colleagues and our recent clinical trial suggest that TNF inhibitors are safe and beneficial in the treatment of patients with cancer and irAEs. We are further assessing these parameters in the second phase of the TICIMEL trial ${ }^{8,9}$.

There is a reply to this letter by Chen. A. Y., Wolchock, J. D. \& Bass, A. R. Nat. Rev. Rheumatol. https://doi.org/10.1038/s41584021-00654-7 (2021).
Anne Montfort ${ }^{1,2}$, Mathieu Virazels ${ }^{1,2,3}$, Céline Colacios ${ }^{1,2,3}$, Nicolas Meyer 1,2,3,4,5凶 and Bruno Ségui (ID 1,2,3,5凶 IINSERM UMR 1037, Cancer Research Center of Toulouse (CRCT), Toulouse, France. ${ }^{2}$ Equipe Labellisée Fondation ARC pour la recherche sur le cancer, Toulouse, France. 3Université Toulouse III - Paul Sabatier, Toulouse, France.

${ }^{4}$ Service d'Oncodermatologie, Institut Universitaire du Cancer (IUCT-O), CHU de Toulouse, Toulouse, France.

${ }^{5}$ These authors contributed equally: Nicolas Meyer, Bruno Ségui. 凶e-mail:meyer.n@chu-toulouse.fr; bruno.segui@ inserm.fr

https://doi.org/10.1038/s41584-021-00653-8

Montfort, A. et al. The TNF paradox in cancer progression and immunotherapy. Front. Immunol. 10, 1818 (2019).

2. Balkwill, F. Tumour necrosis factor and cancer. Nat. Rev. Cancer 9, 361-371 (2009).

3. Chen, A. Y., Wolchok, J. D. \& Bass, A. R. TNF in the era of immune checkpoint inhibitors: friend or foe? Nat. Rev. Rheumatol. 17, 213-223 (2021).

4. Bertrand, F. et al. TNF $\alpha$ blockade overcomes resistance to anti-PD-1 in experimental melanoma. Nat. Commun. 8, 2256 (2017).

5. Perez-Ruiz, E. et al. Prophylactic TNF blockade uncouples efficacy and toxicity in dual CTLA-4 and PD-1 immunotherapy. Nature 569, 428-432 (2019).

6. Lesage, $\mathrm{C}$. et al. Incidence and clinical impact of antiTNF $\alpha$ treatment of severe immune checkpoint inhibitor-induced colitis in advanced melanoma: The Mecolit Survey. J. Immunother. 42, 175-179 (2019).

7. Verheijden, R. J. et al. Association of anti-TNF with decreased survival in steroid refractory ipilimumab and anti-PD 1-treated patients in the Dutch Melanoma Treatment Registry. Clin. Cancer Res. 26, 2268-2274 (2020).

8. Montfort, A. et al. Anti-TNF, a magic bullet in cancer immunotherapy? J. Immunother Cancer 7, 303 (2019). Montfort, A. et al. Combining nivolumab and ipilimumab with infliximab or certolizumab in patients with advanced melanoma: first results of a phase lb clinical trial. Clin. Cancer Res. 27, 1037-1047 (2021).

\section{Competing interests}

B.S. has worked as investigator, consultant and speaker for BMS. N.M. has worked as investigator and/or consultant and/or speaker for BMS, MSD, Roche, Novartis, Pierre Fabre, Amgen, Incyte, Abbvie. B.S. and C.C. have a patent US 10144772B2 issued, a patent WO2015173259A 1 pending, a patent EP3142685B1 issued, a patent ES2748380T3 issued. B.S., C.C. and N.M. have a patent EP3407911A1 pending, a patent JP2019503384A pending, a patent US20190038763A1 pending, and a patent WO2017129790A1 pending. The other authors declare no competing interests.

\title{
Reply to: Combining TNF blockade with immune checkpoint inhibitors in patients with cancer
}

\section{Allen Y. Chen, Jedd D. Wolchok (1) and Anne R. Bass (D)}

We would like to thank Montfort and colleagues for their correspondence (Montfort, A. et al. Combining TNF blockade with immune checkpoint inhibitors in patients with cancer. Nat. Rev. Rheumatol. https://doi.org/10.1038/ s41584-021-00653-8 (2021)) $)^{1}$ on our Review (Chen, A. Y., Wolchok, J. D. \& Bass, A. R. TNF in the era of immune checkpoint inhibitors: friend or foe? Nat. Rev. Rheumatol. 17, 213-223 (2021) $)^{2}$.

We appreciate their highlighting early results of TICIMEL, an open-label phase Ib two-arm study of 14 patients that combined one of two TNF inhibitors, certolizumab or 